\title{
ON THE TIME DECAY OF SOLUTIONS IN MICROPOLAR VISCOELASTICITY
}

\author{
M. CARME LESEDUARTE, ANTONIO MAGAÑA, RAMÓN QUINTANILLA \\ Dept. Matemàtica Aplicada 2, UPC \\ C. Colom 11, 08222 Terrassa, Barcelona, Spain.
}

\begin{abstract}
This paper deals with isotropic micropolar viscoelastic materials. It can be said that that kind of materials have two internal structures: the macrostructure, where the elasticity effects are noticed, and the microstructure, where the polarity of the material points allows them to rotate. We introduce, step by step, dissipation mechanisms in both structures, obtain the corresponding system of equations and determine the behavior of its solutions with respect the time.
\end{abstract}

Keywords: micropolar viscoelasticity, polynomial decay, exponential decay, semigroup of contractions.

\section{INTRODUCTION}

Materials with voids, mixtures of materials or non-simple materials are examples of the socalled "non-classical solids". Many mathematical and mechanical studies have been carried out to understand the behavior of these materials (see, for instance, the book of Ieşan [13]). The first analysis concerning the time decay properties of the solutions for the equations used to describe the behavior of a porous elastic solid was proposed by Quintanilla [30]. The author proved the slow decay of the solutions with respect to the time for elastic-porous materials when there is only one dissipation mechanism in the system: the viscoporosity. After that, a lot of works have been developed to clarify the behavior of the solutions (exponential decay, slow decay, impossibility of localization and/or analyticity) for solids with voids $[4,5,11,12,15,16$, $18,19,20,23,22,24,26,27,28,33]$, for non-simple materials [10, 29] or for mixtures of elastic solids $[1,2,3,31,32]$. Nevertheless, few attention has been paid up to now to micropolar elastic solids. In fact, we only know one contribution of this kind for micropolar elastic solids [21].

The origin of the rational theories about polar continua is attributed to E. and F. Cosserat (see [13] or [8]) at the beginning of the twentieth century. Since then, other contributions on this field have been done: we want to highlight the work of Eringen [7], among others. Nowadays, these materials are a subclass of the micromorphic materials. Metals, polymers, rocks, wood, ceramics, soils, biological materials or pressed powders are typical examples of them.

We can find applications of the micropolar elasticity, for instance, in the works of Elangovan et al. [6] about elastic properties of cellular materials, Fatemi et al. [9] about stress analysis in bone, Kordolemis and Giannakopoulos [14] about smart cables and textiles and Nikogosyan and Sargsyan [25] about thin shells. This list could be enlarged with more contributions, but we think that the cited papers are sufficiently representative.

Date: January 20, 2015. 
In this work we focus on the analysis of qualitative properties of certain one-dimensional deformations for isotropic micropolar viscoelastic materials. That is, materials that, apart from the usual macroscopic movements, allow its material points to rotate. We consider the general system that governs the behavior of a micropolar isotropic viscoelastic solid and we introduce viscosity effects at the macroscopic and microscopic levels. There are three parameters determining the perturbations' damping. We study the relevance of each one separately. Hence, we consider the three possible cases obtained making one of the parameters positive and vanishing the other two. Our aim is to analyze the time decay of the solutions when those dissipation mechanisms are introduced in the system. The first case considers that there is dissipation in the macrostructure (viscoelasticity) but there is not at the microstructure level. In the second case, the dissipation mechanism is considered to be in the microstructure (viscopolarity) but not in the macrostructure. Finally, in the third case we impose dissipation mechanisms in both structures (viscoelasticity and viscopolarity).

The organization of the paper is as follows. In Section 2 we recall the basic equations with the assumptions that we need to set down the problem and we establish the general system that we want to analyze. In Section 3 we focus only on the viscoelasticity, and we notice that the corresponding system is, mathematically, equivalent to a system previously studied in other work. In Section 4 we focus again in only one dissipation mechanism, the viscopolarity, and we prove that the solutions decay in a slow way, moreover, a polynomial rate of decay can be found. In Section 5 we introduce dissipation in both structures: viscoelasticity and viscopolarity, and we show that, then, the solutions are exponentially stable. Section 6 is devoted to the nonlocalization of solutions. Finally, we state the conclusions in Section 7.

\section{BASIC EQUATIONS}

Let us consider an homogeneous isotropic tridimensional micropolar viscoelastic body which occupies a three-dimensional domain $\Gamma$ with a boundary, $\partial \Gamma$, smooth enough to apply the Divergence theorem. We consider the strain measures $e_{i j}$ and $\kappa_{i j}$ which are defined by

$$
e_{i j}=u_{j, i}+\epsilon_{j i k} \phi_{k}, \quad \kappa_{i j}=\phi_{j, i}
$$

where $u_{i}$ are the components of the displacement vector, $\phi_{i}$ are the components of the microrotation and $\epsilon_{j i k}$ is the alternating symbol. In this paper we assume that the stress tensor $t_{i j}$ and the microstress tensor $m_{i j}$ are related to the strain measures $e_{i j}$ and $\kappa_{i j}$ by means of the constitutive equations

$$
\begin{aligned}
& t_{i j}=\lambda e_{r r} \delta_{i j}+(\mu+\sigma) e_{i j}+\mu e_{j i}+\lambda_{v} \dot{e}_{r r} \delta_{i j}+\left(\mu_{v}+\sigma_{v}\right) \dot{e}_{i j}+\mu_{v} \dot{e}_{j i} \\
& m_{i j}=\alpha \kappa_{r r} \delta_{i j}+\beta \kappa_{j i}+\gamma \kappa_{i j}+\alpha_{v} \dot{\kappa}_{r r} \delta_{i j}+\beta_{v} \dot{\kappa}_{j i}+\gamma_{v} \dot{\kappa}_{i j} .
\end{aligned}
$$

Here $\lambda, \mu, \sigma, \lambda_{v}, \mu_{v}, \sigma_{v}, \alpha, \beta, \gamma, \alpha_{v}, \beta_{v}, \gamma_{v}$, are the constitutive coefficients and $\delta_{i j}$ the Kronecker delta.

The evolution equations are

$$
\begin{aligned}
& t_{j i, j}=\rho \ddot{u}_{i}, \\
& \epsilon_{i j k} t_{j k}+m_{j i, j}=J \ddot{\phi}_{i},
\end{aligned}
$$

where $\rho$ and $J$ are positive constants whose physical meaning is well known.

As the material is isotropic, the system of the field equations is given by

$$
\left\{\begin{array}{l}
\rho \ddot{u}_{i}=(\mu+\sigma) \Delta u_{i}+(\lambda+\mu) u_{r, r i}+\sigma \epsilon_{i r s} \phi_{s, r}+\left(\mu_{v}+\sigma_{v}\right) \Delta \dot{u}_{i}+\left(\lambda_{v}+\mu_{v}\right) \dot{u}_{r, r i}+\sigma_{v} \epsilon_{i r s} \dot{\phi}_{s, r} \\
J \ddot{\phi}_{i}=\gamma \Delta \phi_{i}+(\alpha+\beta) \phi_{r, r i}+\sigma \epsilon_{i r s} u_{s, r}-2 \sigma \phi_{i}+\gamma_{v} \Delta \dot{\phi}_{i}+\left(\alpha_{v}+\beta v\right) \dot{\phi}_{r, r i}+\sigma_{v} \epsilon_{i r s} \dot{u}_{s, r}-2 \sigma_{v} \dot{\phi}_{i} .
\end{array}\right.
$$


Here $\Delta$ means the three-dimensional Laplace operator.

Our aim is to analyze solutions corresponding to certain one-dimensional deformations. For this reason, we impose solutions of the form $u_{1}=u_{2}=0, u_{3}=u\left(x_{2}\right), \phi_{2}=\phi_{3}=0$ and $\phi_{1}=\phi\left(x_{2}\right)$. To simplify, we will write $x=x_{2}$. Then, the above system reduces to

$$
\left\{\begin{array}{l}
\rho \ddot{u}=(\mu+\sigma) u_{x x}-\sigma \phi_{x}+\left(\mu_{\nu}+\sigma_{\nu}\right) \dot{u}_{x x}-\sigma_{\nu} \dot{\phi}_{x} \\
J \ddot{\phi}=\gamma \phi_{x x}+\sigma u_{x}-2 \sigma \phi+\gamma_{\nu} \dot{\phi}_{x x}+\sigma_{\nu} \dot{u}_{x}-2 \sigma_{\nu} \dot{\phi}
\end{array}\right.
$$

As usual, in our work we will assume that $\rho>0, J>0, \gamma>0$ and $\mu+\sigma>0$. We also assume that $2(\sigma+\mu) \sigma>\sigma^{2}$. The last condition implies $\sigma>0$.

Attending to the dissipative terms, we will analyze three different cases.

(1) $\sigma_{\nu}=0, \gamma_{\nu}=0$ and $\mu_{\nu}>0$.

(2) $\sigma_{\nu}=0, \gamma_{\nu}>0$ and $\mu_{\nu}=0$.

(3) $\sigma_{\nu}>0, \gamma_{\nu}=0$ and $\mu_{\nu}=0$.

Notice that if solutions of the form $u_{1}=u_{2}=0, u_{3}=u\left(x_{1}\right), \phi_{1}=\phi_{3}=0$ and $\phi_{2}=\phi\left(x_{1}\right)$ are imposed, then an analogous system is obtained.

\section{First CASE: Viscoelasticity}

Our aim is to determine the asymptotic behavior of the solutions of system (2.2) with respect to the time when coefficients $\sigma_{\nu}$ and $\gamma_{\nu}$ are supposed to be 0 and $\mu_{\nu}$ is strictly positive. Physically, that means that there is dissipation at the macrostructure but there is not at the microstructure level.

Taking these conditions into account, system (2.2) becomes

$$
\left\{\begin{array}{l}
\rho \ddot{u}=(\mu+\sigma) u_{x x}-\sigma \phi_{x}+\mu_{\nu} \dot{u}_{x x} \\
J \ddot{\phi}=\gamma \phi_{x x}+\sigma u_{x}-2 \sigma \phi
\end{array}\right.
$$

To have a well posed problem we need to impose boundary and initial conditions. Thus, we assume that the solutions satisfy the boundary conditions

$$
u(0, t)=u(\pi, t)=\phi_{x}(0, t)=\phi_{x}(\pi, t)=0,
$$

and the initial conditions

$$
u(x, 0)=u_{0}(x), \quad \dot{u}(x, 0)=v_{0}(x), \quad \phi(x, 0)=\phi_{0}(x), \quad \dot{\phi}(x, 0)=\varphi_{0}(x) .
$$

There are solutions (uniform in the variable $x$ ) that do not decay. To avoid these cases, we will also assume that

$$
\int_{0}^{\pi} \phi_{0}(x) d x=\int_{0}^{\pi} \varphi_{0}(x) d x=0 .
$$

With the above assumptions, it is not difficult to prove that the solutions of the problem determined by (3.1)-(3.3) decay in a slow way, or, in other words, that a uniform rate of decay of exponential type for all the solutions cannot be obtained. We omit the proof because this system, with the boundary and initial conditions mentioned above, corresponds to a subclass of the system studied by the authors in a previous work [19] (it is also system (3.1) of the cited paper with some changes in the names of the constitutive constants). Nevertheless, the physical contexts are different. In fact, a polynomial rate of decay for the solutions can be found (see [23], page 1299). 


\section{SECOND CASE: VISCOPOLARITY}

Now we consider that $\sigma_{\nu}$ and $\mu_{\nu}$ vanish and $\gamma_{\nu}$ is strictly positive. In this case, the dissipation mechanism only affects the microstructure. Therefore, system (2.2) reduces to

$$
\left\{\begin{array}{l}
\rho \ddot{u}=(\mu+\sigma) u_{x x}-\sigma \phi_{x} \\
J \ddot{\phi}=\gamma \phi_{x x}+\sigma u_{x}-2 \sigma \phi+\gamma_{\nu} \dot{\phi}_{x x}
\end{array}\right.
$$

We impose the same boundary and initial conditions as in the previous section.

Theorem 4.1. Let $(u, \phi)$ be a solution of the problem determined by system (4.1), boundary conditions (3.2) and initial conditions (3.3). Then $(u, \phi)$ decays in a slow way.

Proof. We will see that there exists a solution of the system (4.1) of the form

$$
u=K_{1} e^{\omega t} \sin (n x), \quad \phi=K_{2} e^{\omega t} \cos (n x),
$$

such that $\Re(\omega)>-\epsilon$ for all positive $\epsilon$. Hence, $\omega$ as near as desired to the imaginary axis can be found. This fact proves that it is impossible to have uniform exponential decay for the solutions of the system.

Suppose that $u$ and $\phi$ are as above. Then, replacing them in (4.1) the following linear and homogeneous system in the unknowns $K_{1}$ and $K_{2}$ is obtained:

$$
\left(\begin{array}{cc}
(\mu+\sigma) n^{2}+\rho \omega^{2} & -n \sigma \\
-n \sigma & \left(\gamma+\gamma_{\nu} \omega\right) n^{2}+J \omega^{2}+2 \sigma
\end{array}\right)\left(\begin{array}{l}
K_{1} \\
K_{2}
\end{array}\right)=\left(\begin{array}{l}
0 \\
0
\end{array}\right)
$$

This linear system will have nontrivial solutions if, and only if, the determinant of the coefficients matrix is equal to zero. Let $p(x)$ be this determinant once $\omega$ is replaced by $x$ :

$p(x)=J \rho x^{4}+n^{2} \gamma_{\nu} \rho x^{3}+\left(J n^{2}(\mu+\sigma)+n^{2} \gamma \rho+2 \rho \sigma\right) x^{2}+\gamma_{\nu}(\mu+\sigma) n^{4} x+n^{4} \gamma(\mu+\sigma)+n^{2} \sigma(2 \mu+\sigma)$.

It is a fourth degree polynomial and, hence, its roots can be computed by formula. Nevertheless, the expressions of the roots that we have found using Mathematica are too complicated to enable us decide whether their real parts are negative or not. To prove that $p(x)$ has roots as near as desired to the imaginary axis, we will show that for any $\epsilon>0$ there are roots of $p(x)$ located at the right side of the vertical line $\Re(z)=-\epsilon$, or, equivalently, that the polynomial $p(x-\epsilon)$ has a root with positive real part. To prove that, we use the Routh-Hurwitz theorem. It assesses that, if $a_{0}>0$, then all the roots of a polynomial

$$
a_{0} x^{4}+a_{1} x^{3}+a_{2} x^{2}+a_{3} x+a_{4},
$$

have negative real part if, and only if, all the leading minors of matrix

$$
\left(\begin{array}{cccc}
a_{1} & a_{0} & 0 & 0 \\
a_{3} & a_{2} & a_{1} & a_{0} \\
0 & a_{4} & a_{3} & a_{2} \\
0 & 0 & 0 & a_{4}
\end{array}\right)
$$

are positive. We denote by $L_{i}$, for $i=1,2,3,4$, the leading minors of this matrix.

In our case, direct computations give that $L_{3}$ is an eight degree polynomial on $n$ :

$$
L_{3}=-2 \gamma_{\nu}^{3} \epsilon \rho^{2}(\mu+\sigma) n^{8}+R(n)
$$

where $R(n)$ is a polynomial on $n$ of degree 6 . Thus, for $n$ large enough, $L_{3}$ is negative and $p(x-\epsilon)$ has at least one root with positive real part. 
We now try to find a polynomial rate of decay for the solutions. And we begin by noting that the solutions of this problem can be generated by means of a semigroup of contractions.

If we denote $v=\dot{u}, \varphi=\dot{\phi}$ and $D=\frac{d}{d x}$, we can restate system (4.1) in the following way:

$$
\left\{\begin{array}{l}
\dot{u}=v \\
\dot{v}=\frac{1}{\rho}\left[(\mu+\sigma) D^{2} u-\sigma D \phi\right] \\
\dot{\phi}=\varphi \\
\dot{\varphi}=\frac{1}{J}\left[\gamma D^{2} \phi+\sigma D u-2 \sigma \phi+\gamma_{\nu} D^{2} \varphi\right]
\end{array}\right.
$$

Therefore, the semigroup of contractions is defined in the Hilbert space

$$
\mathcal{H}=\left\{(u, v, \phi, \varphi) \in H_{0}^{1} \times L^{2} \times H^{1} \times L^{2}, \int_{0}^{\pi} \phi(x) d x=\int_{0}^{\pi} \varphi(x) d x=0\right\}
$$

by the operator

$$
\mathcal{A}=\left(\begin{array}{cccc}
0 & I & 0 & 0 \\
\frac{\mu+\sigma}{\rho} D^{2} & 0 & -\frac{\sigma}{\rho} D & 0 \\
0 & 0 & 0 & I \\
\frac{\sigma}{J} D & 0 & \frac{\gamma D^{2}-2 \sigma}{J} & -\frac{\gamma_{\nu}}{J} D^{2}
\end{array}\right)
$$

where $I$ denotes the identity operator.

And, if $U=(u, v, \phi, \varphi)$, then our initial-boundary value problem can be written as

$$
\frac{d U}{d t}=\mathcal{A} U, U(0)=U_{0}=\left(u_{0}, v_{0}, \phi_{0}, \varphi_{0}\right)
$$

Now, we define an inner product in $\mathcal{H}$. If $U^{*}=\left(u^{*}, v^{*}, \phi^{*}, \varphi^{*}\right)$, then

$$
\left\langle U, U^{*}\right\rangle=\int_{0}^{\pi}\left(\rho v \bar{v}^{*}+J \varphi \bar{\varphi}^{*}+(\mu+\sigma) u_{x} \bar{u}_{x}^{*}+\gamma \phi_{x} \bar{\phi}_{x}^{*}+2 \sigma \phi \bar{\phi}^{*}-\sigma\left(u_{x} \bar{\phi}^{*}+\bar{u}_{x}^{*} \phi\right)\right) d x .
$$

Here a superposed bar denotes the conjugate complex number. It is worth recalling that this product is equivalent to the usual product in the Hilbert space $\mathcal{H}$.

The domain of $\mathcal{A}$ is

$$
\mathcal{D}(\mathcal{A})=\left\{U \in \mathcal{H}: u \in H^{2}, v \in H_{0}^{1} \cap H^{2}, \phi \in H^{2}, D \phi \in H_{0}^{1}, \varphi \in H^{2}\right\} .
$$

Notice that $\mathcal{D}(\mathcal{A})$ is dense in $\mathcal{H}$.

We will show that the solutions of

$$
\frac{d U}{d t}=\mathcal{A} U, \quad U(0)=U_{0} \in \mathcal{D}(\mathcal{A})
$$

decays polynomially to zero.

Our starting point is to define the first order energy:

$$
E_{1}(t)=\frac{1}{2} \int_{0}^{\pi}\left(\rho|\dot{u}|^{2}+J|\dot{\phi}|^{2}+(\mu+\sigma) u_{x}^{2}+\gamma \phi_{x}^{2}+2 \sigma \phi^{2}-2 \sigma u_{x} \phi\right) d x .
$$

We introduce also the second order energies: $E_{2}(t)=E_{1}(t, \dot{u}, \dot{\phi})$ and $E_{3}(t)=E_{1}\left(t, u_{x}, \phi_{x}\right)$.

Direct computations give

$$
\frac{d E_{1}}{d t}=-\gamma_{\nu} \int_{0}^{\pi}\left|\dot{\phi}_{x}\right|^{2} d x, \quad \frac{d E_{2}}{d t}=-\gamma_{\nu} \int_{0}^{\pi}\left|\ddot{\phi}_{x}\right|^{2} d x, \quad \frac{d E_{3}}{d t}=-\gamma_{\nu} \int_{0}^{\pi}\left|\dot{\phi}_{x x}\right|^{2} d x .
$$


Let us define the following functionals:

$$
\begin{aligned}
& F(t)=\int_{0}^{\pi}\left(J \phi_{x} \dot{\phi}_{x}+\frac{\gamma_{\nu}}{2} \phi_{x x}^{2}\right) d x, \\
& G(t)=-\int_{0}^{\pi} \rho \dot{u} \phi_{x} d x .
\end{aligned}
$$

Lemma 4.2. With the above notations, if $\left(u_{0}, v_{0}, \phi_{0}, \varphi_{0}\right) \in \mathcal{D}(\mathcal{A})$, then the corresponding solution of system (4.1) satisfies

$$
\frac{d}{d t}\left(F(t)+\frac{\sigma}{\mu+\sigma} G(t)\right) \leq-\gamma \int_{0}^{\pi} \phi_{x x}^{2} d x-\frac{\sigma^{2}+2 \mu \sigma}{\mu+\sigma} \int_{0}^{\pi} \phi_{x}^{2} d x+\epsilon \int_{0}^{\pi}|\dot{u}|^{2} d x+c_{\epsilon} \int_{0}^{\pi}\left|\dot{\phi}_{x}\right|^{2} d x
$$

where $\epsilon$ is a positive but small real number and $c_{\epsilon}$ is a computable positive real number that depends on $\epsilon$.

Proof. First we differentiate functional $F(t)$ with respect to the time:

$$
\begin{aligned}
\frac{d}{d t} F(t) & =\int_{0}^{\pi}\left(J \dot{\phi}_{x} \dot{\phi}_{x}+J \phi_{x} \ddot{\phi}_{x}+\gamma_{\nu} \phi_{x x} \dot{\phi}_{x x}\right) d x \\
& =J \int_{0}^{\pi}\left|\dot{\phi}_{x}\right|^{2} d x-\int_{0}^{\pi} \phi_{x x} J \ddot{\phi} d x+\gamma_{\nu} \int_{0}^{\pi} \phi_{x x} \dot{\phi}_{x x} d x .
\end{aligned}
$$

In the last expression we use the first equation of system (4.1) and we get:

$$
\begin{aligned}
\frac{d}{d t} F(t) & =J \int_{0}^{\pi}\left|\dot{\phi}_{x}\right|^{2} d x-\int_{0}^{\pi} \phi_{x x}\left(\gamma \phi_{x x}+\sigma u_{x}-2 \sigma \phi+\gamma_{\nu} \dot{\phi}_{x x}\right) d x+\gamma_{\nu} \int_{0}^{\pi} \phi_{x x} \dot{\phi}_{x x} d x \\
& =J \int_{0}^{\pi}\left|\dot{\phi}_{x}\right|^{2} d x-\gamma \int_{0}^{\pi} \phi_{x x}^{2} d x-\sigma \int_{0}^{\pi} u_{x} \phi_{x x} d x-2 \sigma \int_{0}^{\pi} \phi_{x}^{2} d x .
\end{aligned}
$$

And now we differentiate the functional $G(t)$. In the obtained expression we take into account the second equation of system (4.1). After some computations we get

$$
\frac{d}{d t} G(t)=(\mu+\sigma) \int_{0}^{\pi} u_{x} \phi_{x x} d x+\sigma \int_{0}^{\pi} \phi_{x}^{2} d x-\rho \int_{0}^{\pi} \dot{u} \dot{\phi}_{x} d x .
$$

Finally, we consider the new functional $F+\frac{\sigma}{\mu+\sigma} G$. If we take into account (4.5) and (4.6), we obtain

$$
\frac{d}{d t}\left(F(t)+\frac{\sigma}{\mu+\sigma} G(t)\right)=J \int_{0}^{\pi}\left|\dot{\phi}_{x}\right|^{2} d x-\gamma \int_{0}^{\pi} \phi_{x x}^{2} d x-\frac{\sigma^{2}+2 \mu \sigma}{\mu+\sigma} \int_{0}^{\pi} \phi_{x}^{2} d x-\frac{\rho \sigma}{\mu+\sigma} \int_{0}^{\pi} \dot{u} \dot{\phi}_{x} d x .
$$

Notice that from our hypothesis $2(\mu+\sigma) \sigma>\sigma^{2}$, we also obtain $2 \mu \sigma+\sigma^{2}>0$.

From the last equality, taking into account that for all $\epsilon>0$

$$
\int_{0}^{\pi} \dot{u} \dot{\phi}_{x} d x \leq \frac{\epsilon}{2} \int_{0}^{\pi}|\dot{u}|^{2} d x+\frac{1}{2 \epsilon} \int_{0}^{\pi}\left|\dot{\phi}_{x}\right|^{2} d x
$$

the inequality of the lemma follows. 
We introduce two more functionals:

$$
\begin{aligned}
& S(t)=-\int_{0}^{\pi} J \dot{\phi} u_{x} d x, \\
& T(t)=\int_{0}^{\pi} \rho \dot{u} u d x .
\end{aligned}
$$

Lemma 4.3. With the same hypotheses of Lemma 4.2 we have

$$
\begin{array}{r}
\frac{d}{d t}\left(S(t)-\frac{\sigma}{8(\mu+\sigma+\epsilon)} T(t)\right) \leq-\frac{\sigma}{4} \int_{0}^{\pi}\left|u_{x}\right|^{2} d x-\frac{\rho \sigma}{4(\mu+\sigma+\epsilon)} \int_{0}^{\pi}|\dot{u}|^{2} d x \\
+C_{\epsilon}\left(\int_{0}^{\pi}\left|\phi_{x x}\right|^{2} d x+\int_{0}^{\pi}\left|\dot{\phi}_{x x}\right|^{2} d x\right)
\end{array}
$$

where $\epsilon$ is a positive but small real number and $C_{\epsilon}$ is a computable positive real number depending on $\epsilon$.

Proof. We compute the following derivative

$$
\frac{d}{d t} \int_{0}^{\pi} J \dot{\phi} u_{x} d x=\int_{0}^{\pi} J \ddot{\phi} u_{x} d x+\int_{0}^{\pi} J \dot{\phi} \dot{u}_{x} d x
$$

Now we use the second equation of the system (4.1):

$$
\begin{aligned}
\frac{d}{d t} \int_{0}^{\pi} J \dot{\phi} u_{x} d x & =\int_{0}^{\pi}\left(\gamma \phi_{x x}+\sigma u_{x}-2 \sigma \phi+\gamma_{\nu} \dot{\phi}_{x x}\right) u_{x} d x+J \int_{0}^{\pi} \dot{\phi} \dot{u}_{x} d x \\
& =\sigma \int_{0}^{\pi} u_{x}^{2} d x+\gamma \int_{0}^{\pi} \phi_{x x} u_{x} d x-2 \sigma \int_{0}^{\pi} \phi u_{x} d x+\gamma_{\nu} \int_{0}^{\pi} \dot{\phi}_{x x} u_{x} d x+J \int_{0}^{\pi} \dot{\phi} \dot{u}_{x} d x
\end{aligned}
$$

Therefore, recalling the definition of $S(t)$ and using Poincaré inequality, we get

$$
\frac{d}{d t} S(t) \leq-\frac{\sigma}{2} \int_{0}^{\pi}\left|u_{x}\right|^{2} d x+C\left(\int_{0}^{\pi}\left|\phi_{x x}\right|^{2} d x+\int_{0}^{\pi}\left|\dot{\phi}_{x x}\right|^{2} d x\right)+J \int_{0}^{\pi} \dot{\phi}_{x} \dot{u} d x
$$

where $C$ is a positive computable constant.

We also know that

$$
\int_{0}^{\pi} \dot{\phi}_{x} \dot{u} d x \leq \frac{\rho \sigma}{8(\mu+\sigma+\epsilon)} \int_{0}^{\pi}|\dot{u}|^{2} d x+C_{1} \int_{0}^{\pi}\left|\dot{\phi}_{x}\right|^{2} d x
$$

And, applying the Poincaré inequality,

$$
\int_{0}^{\pi} \dot{\phi}_{x} \dot{u} d x \leq \frac{\rho \sigma}{8(\mu+\sigma+\epsilon)} \int_{0}^{\pi}|\dot{u}|^{2} d x+C_{2} \int_{0}^{\pi}\left|\dot{\phi}_{x x}\right|^{2} d x .
$$


Analogously,

$$
\begin{aligned}
\frac{d}{d t} T(t) & =\int_{0}^{\pi} \rho \ddot{u} u d x+\int_{0}^{\pi} \rho \dot{u} \dot{u} d x \\
& =\rho \int_{0}^{\pi}|\dot{u}|^{2} d x+\int_{0}^{\pi}\left((\mu+\sigma) u_{x x}-\sigma \phi_{x}\right) u d x \\
& =\rho \int_{0}^{\pi}|\dot{u}|^{2} d x-(\mu+\sigma) \int_{0}^{\pi} u_{x}^{2} d x-\sigma \int_{0}^{\pi} \phi_{x} u d x \\
& \geq \rho \int_{0}^{\pi}|\dot{u}|^{2} d x-(\mu+\sigma) \int_{0}^{\pi} u_{x}^{2} d x-\epsilon \int_{0}^{\pi}|u|^{2} d x-c \int_{0}^{\pi}\left|\phi_{x}\right|^{2} d x \\
& \geq \rho \int_{0}^{\pi}|\dot{u}|^{2} d x-(\mu+\sigma+\epsilon) \int_{0}^{\pi}\left|u_{x}\right|^{2} d x-c \int_{0}^{\pi}\left|\phi_{x}\right|^{2} d x
\end{aligned}
$$

Finally, writing the linear combination of $S(t)$ and $T(t)$, the inequality of the lemma follows.

Theorem 4.4. If $\left(u_{0}, v_{0}, \phi_{0}, \varphi_{0}\right) \in \mathcal{D}(\mathcal{A})$, then there exists a positive constant $C$ such that

$$
E_{1}(t) \leq \frac{C\left\|\left(u_{0}, v_{0}, \phi_{0}, \varphi_{0}\right)\right\|_{\mathcal{D}(\mathcal{A})}^{2}}{t}
$$

Moreover, if $\left(u_{0}, v_{0}, \phi_{0}, \varphi_{0}\right) \in \mathcal{D}\left(\mathcal{A}^{\alpha}\right)$, then

$$
E_{1}(t) \leq \frac{C_{\alpha}\left\|\left(u_{0}, v_{0}, \phi_{0}, \varphi_{0}\right)\right\|_{\mathcal{D}\left(\mathcal{A}^{\alpha}\right)}^{2}}{t^{\alpha}} .
$$

Proof. We introduce a new functional $L(t)$ as a linear combination of previous functions:

$$
L(t)=S(t)-\frac{\sigma}{8(\mu+\sigma+\epsilon)} T(t)+K_{0}\left(F(t)+\frac{\sigma}{\mu+\sigma} G(t)\right)+K_{1} E_{1}(t)+K_{2} E_{2}(t)+K_{3} E_{3}(t),
$$

where $K_{i}$, for $i=0,1,2,3$, are large enough to make $L(t) \geq 0$. Then, from Lemmas 4.2 and 4.3 it can be shown that for $K_{i}$ large enough we have

$$
\frac{d}{d t} L(t) \leq-K_{\sigma} E_{1}(t)
$$

where $K_{\sigma}$ is a computable positive constant.

Therefore, integrating from 0 to $t$ we obtain

$$
L(t)+K_{\sigma} \int_{0}^{t} E_{1}(s) d s \leq L(0) .
$$

On the other side, we know that

$$
\frac{d}{d t}\left(t E_{1}(t)\right)=E_{1}(t)+t \frac{d}{d t} E_{1}(t) \leq E_{1}(t) .
$$

Finally, a quadrature gives

$$
t E_{1}(t) \leq \int_{0}^{t} E_{1}(s) d s \leq \frac{1}{K_{\sigma}} L(0)
$$

And this fact implies the polynomial decay.

Remark: From a mathematical point of view, system (4.1) is quite near to system

$$
\left\{\begin{array}{l}
\rho \ddot{u}=(\mu+\sigma) u_{x x}-\sigma \phi_{x} \\
J \ddot{\phi}=\gamma \phi_{x x}+\sigma u_{x}-2 \sigma \phi-\tau \dot{\phi}
\end{array}\right.
$$


where $\tau$ is a positive real number. With the same initial and boundary conditions that those of system (4.1), it can be shown that the solution of this system decay is a slow way [30]. But to prove this result it is necessary to have $\gamma \rho \neq J(\mu+\sigma)$. In the case we have analyzed, although the dissipation mechanism is (intuitively) stronger, we do not need any relation among the constitutive coefficients of the system to obtain the slow decay.

An analogous remark applies to system (3.1) when $\dot{u}_{x x}$ is replaced by $\dot{u}$.

\section{THIRD CASE: VISCOELASTICITY AND VISCOPOLARITY}

Finally, we consider that $\gamma_{\nu}$ and $\mu_{\nu}$ are 0 and $\sigma_{\nu}$ is strictly positive. In this case, there are two dissipation mechanisms, one at the macrostructure level and another one at the microstructure. Therefore, system (2.2) becomes

$$
\left\{\begin{array}{l}
\rho \ddot{u}=(\mu+\sigma) u_{x x}-\sigma \phi_{x}+\sigma_{\nu} \dot{u}_{x x}-\sigma_{\nu} \dot{\phi}_{x} \\
J \ddot{\phi}=\gamma \phi_{x x}+\sigma u_{x}-2 \sigma \phi+\sigma_{\nu} \dot{u}_{x}-2 \sigma_{\nu} \dot{\phi}
\end{array}\right.
$$

As in the previous sections, we impose boundary conditions (3.2) and initial conditions (3.3).

We will prove that the solutions of the system (5.1) that satisfies (3.2)-(3.3) decay exponentially. To do so, we will use the semigroup argument again.

System (5.1) can be written in the following way:

$$
\left\{\begin{array}{l}
\dot{u}=v \\
\dot{v}=\frac{1}{\rho}\left[(\mu+\sigma) D^{2} u+\sigma_{\nu} D^{2} v-\sigma D \phi-\sigma_{\nu} D \varphi\right] \\
\dot{\phi}=\varphi \\
\dot{\varphi}=\frac{1}{J}\left[\gamma D^{2} \phi+\sigma D u-2 \sigma \phi+\sigma_{\nu} D v-2 \sigma_{\nu} \varphi\right]
\end{array}\right.
$$

And, if $U=(u, v, \phi, \varphi)$, then our initial-boundary value problem (abusing a little bit the notation of the paper) is

$$
\frac{d U}{d t}=\mathcal{A} U, \quad U_{0}=\left(u_{0}, v_{0}, \phi_{0}, \varphi_{0}\right)
$$

where $\mathcal{A}$ is now the following $4 \times 4$ matrix:

$$
\mathcal{A}=\left(\begin{array}{cccc}
0 & I & 0 & 0 \\
\frac{\mu+\sigma}{\rho} D^{2} & \frac{\sigma_{\nu}}{\rho} D^{2} & -\frac{\sigma}{\rho} D & -\frac{\sigma_{\nu}}{\rho} D \\
0 & 0 & 0 & I \\
\frac{\sigma}{J} D & \frac{\sigma_{\nu}}{J} D & \frac{\gamma D^{2}-2 \sigma}{J} & -\frac{2 \sigma_{\nu}}{J}
\end{array}\right) .
$$

It can be proved that the general solutions of system (5.1) are given by the semigroup of contractions generated by the operator $\mathcal{A}$.

Direct calculation gives

$$
\Re\langle\mathcal{A} U, U\rangle=-\int_{0}^{\pi}\left(\sigma_{\nu}\left(\|D v\|^{2}-2 \Re(\varphi, D v)+\|\varphi\|^{2}\right)+\sigma_{\nu}\|\varphi\|^{2}\right) d x \leq 0 .
$$

To show the exponential stability we use a result due to Gearhart (Liu and Zheng, 1999 [17]) which states that a semigroup of contractions on a Hilbert space is exponentially stable if and only if

$$
\{i \lambda, \lambda \text { is real }\} \text { is contained in the resolvent of } \mathcal{A} \text {, }
$$


and

$$
\varlimsup_{|\lambda| \rightarrow \infty}\left\|(i \lambda \mathcal{I}-\mathcal{A})^{-1}\right\|<\infty
$$

where $\mathcal{I}$ denotes the identity operator.

To prove these conditions we need first the following result.

Lemma 5.1. Let $\mathcal{A}$ be the above defined matrix. Then, 0 is in the resolvent of $\mathcal{A}$.

Proof: For any $\mathcal{F}=\left(f_{1}, f_{2}, f_{3}, f_{4}\right) \in \mathcal{H}$ we will find $U \in \mathcal{H}$ such that $\mathcal{A} U=\mathcal{F}$, or equivalently:

$$
\left.\begin{array}{rl}
v & =f_{1} \\
\frac{1}{\rho}\left[(\mu+\sigma) D^{2} u+\sigma_{\nu} D^{2} v-\sigma D \phi-\sigma_{\nu} D \varphi\right] & =f_{2} \\
\varphi & =f_{3} \\
\frac{1}{J}\left[\gamma D^{2} \phi+\sigma D u-2 \sigma \phi+\sigma_{\nu} D v-2 \sigma_{\nu} \varphi\right] & =f_{4}
\end{array}\right\}
$$

Therefore, the second and fourth equations can be written in terms of $f_{1}$ and $f_{3}$ as follows:

$$
\left.\begin{array}{c}
(\mu+\sigma) D^{2} u-\sigma D \phi=\rho f_{2}-\sigma_{\nu} D^{2} f_{1}+\sigma_{\nu} D f_{3} \\
\sigma D u+\gamma D^{2} \phi-2 \sigma \phi=J f_{4}-\sigma_{\nu} D f_{1}+2 \sigma_{\nu} f_{3}
\end{array}\right\}
$$

To prove the solvability of this system we take $f_{1}=\sum_{n=1}^{\infty} a_{n} \sin (n x), f_{2}=\sum_{n=1}^{\infty} b_{n} \sin (n x)$, $f_{3}=\sum_{n=1}^{\infty} c_{n} \cos (n x)$ and $f_{4}=\sum_{n=1}^{\infty} d_{n} \cos (n x)$ and we want to show that it is possible to find $u=\sum_{n=1}^{\infty} u_{n} \sin (n x)$ and $\phi=\sum_{n=1}^{\infty} \phi_{n} \cos (n x)$ such that $\sum_{n=1}^{\infty} n^{2} u_{n}^{2}<\infty$ and $\sum_{n=1}^{\infty} \phi_{n}^{2}<\infty$. From the hypotheses we know that $\sum_{n=1}^{\infty} n^{2} a_{n}^{2}<\infty, \sum_{n=1}^{\infty} b_{n}^{2}<\infty, \sum_{n=1}^{\infty} n^{2} c_{n}^{2}<\infty$ and $\sum_{n=1}^{\infty} d_{n}^{2}<\infty$.

Substituting the above expressions in system (5.7) and simplifying we get a linear system in the unknowns $u_{n}$ and $\phi_{n}$ for each $n$. We have used Mathematica to solve this system. The solution is

$$
\begin{aligned}
& u_{n}=-\frac{a_{n} n^{2} \sigma_{\nu}\left(\gamma n^{2}+\sigma\right)+b_{n} \rho\left(\gamma n^{2}+2 \sigma\right)-c_{n} \gamma n^{3} \sigma_{\nu}+d_{n} J n \sigma}{n^{2}\left(\sigma(2 \mu+\sigma)+\gamma n^{2}(\mu+\sigma)\right)}, \\
& \phi_{n}=-\frac{-a_{n} \mu n^{2} \sigma_{\nu}+b_{n} \rho \sigma+c_{n} n \sigma_{\nu}(2 \mu+\sigma)+d_{n} J n(\mu+\sigma)}{n\left(\sigma(2 \mu+\sigma)+\gamma n^{2}(\mu+\sigma)\right)} .
\end{aligned}
$$

Thus, it is clear that $u_{n}$ and $\phi_{n}$ satisfy the desired conditions.

It is also clear from the calculations that

$$
\|U\|_{\mathcal{H}} \leq K\|\mathcal{F}\|_{\mathcal{H}}
$$

where $K$ is a constant independent of $U$.

Lemma 5.2. Let $\mathcal{A}$ be the same matrix as in Lemma 5.1. Then condition (5.4) is satisfied.

Proof: We split the proof in three steps.

(i) Since 0 is in the resolvent of $\mathcal{A}$, by the contraction mapping theorem, for any real $\lambda$ such that $|\lambda|<\left\|\mathcal{A}^{-1}\right\|^{-1}$, the operator $i \lambda \mathcal{I}-\mathcal{A}=\mathcal{A}\left(i \lambda \mathcal{A}^{-1}-\mathcal{I}\right)$ is invertible. Moreover, $\left\|(i \lambda \mathcal{I}-\mathcal{A})^{-1}\right\|$ is a continuous function of $\lambda$ in the interval $\left(-\left\|\mathcal{A}^{-1}\right\|^{-1},\left\|\mathcal{A}^{-1}\right\|^{-1}\right)$.

(ii) If $\sup \left\{\left\|(i \lambda \mathcal{I}-\mathcal{A})^{-1}\right\|,|\lambda|<\left\|\mathcal{A}^{-1}\right\|^{-1}\right\}=M<\infty$, then, using the contraction theorem again, the operator

$$
i \lambda \mathcal{I}-\mathcal{A}=\left(i \lambda_{0} \mathcal{I}-\mathcal{A}\right)\left(\mathcal{I}+i\left(\lambda-\lambda_{0}\right)\left(i \lambda_{0} \mathcal{I}-\mathcal{A}\right)^{-1}\right),
$$

is invertible for $\left|\lambda-\lambda_{0}\right|<M^{-1}$. Hence, choosing $\lambda_{0}$ close enough to $\left\|\mathcal{A}^{-1}\right\|^{-1}$, the set $\left\{\lambda,|\lambda|<\left\|\mathcal{A}^{-1}\right\|^{-1}+M^{-1}\right\}$ is contained in the resolvent of $\mathcal{A}$ and $\left\|(i \lambda \mathcal{I}-\mathcal{A})^{-1}\right\|$ is a continuous function of $\lambda$ in the interval $\left(-\left\|\mathcal{A}^{-1}\right\|^{-1}-M^{-1},\left\|\mathcal{A}^{-1}\right\|^{-1}+M^{-1}\right)$. 
(iii) Suppose that the statement of this lemma is not true. Then, there exists a real number $\sigma \neq 0$ with $\left\|\mathcal{A}^{-1}\right\|^{-1} \leq|\sigma|<\infty$ satisfying that the set $\{i \lambda,|\lambda|<|\sigma|\}$ is in the resolvent of $\mathcal{A}$ and $\sup \left\{\left\|(i \lambda \mathcal{I}-\mathcal{A})^{-1}\right\|,|\lambda|<|\sigma|\right\}=\infty$. In this case, we can find a sequence of real numbers, $\lambda_{n}$, with $\lambda_{n} \rightarrow \sigma,\left|\lambda_{n}\right|<|\sigma|$, and a sequence of unit norm vectors in the domain of $\mathcal{A}, U_{n}=\left(u_{n}, v_{n}, \phi_{n}, \varphi_{n}\right)$, such that

$$
\left\|\left(i \lambda_{n} \mathcal{I}-\mathcal{A}\right) U_{n}\right\| \rightarrow 0
$$

Writing this condition term by term we get

$$
\begin{gathered}
i \lambda_{n} u_{n}-v_{n} \rightarrow 0 \text { in } H^{1}, \\
i \lambda_{n} v_{n}-\frac{1}{\rho}\left[(\mu+\sigma) D^{2} u_{n}+\sigma_{\nu} D^{2} v_{n}-\sigma D \phi_{n}-\sigma_{\nu} D \varphi_{n}\right] \rightarrow 0 \text { in } L^{2}, \\
i \lambda_{n} \phi_{n}-\varphi_{n} \rightarrow 0 \text { in } H^{1}, \\
i \lambda_{n} \varphi_{n}-\frac{1}{J}\left[\gamma D^{2} \phi_{n}+\sigma D u_{n}-2 \sigma \phi_{n}+\sigma_{\nu} D v_{n}-2 \sigma_{\nu} \varphi_{n}\right] \rightarrow 0 \text { in } L^{2},
\end{gathered}
$$

Taking the inner product of $\left(i \lambda_{n} \mathcal{I}-\mathcal{A}\right) U_{n}$ times $U_{n}$ in $\mathcal{H}$ using (5.3) and selecting its real part we obtain $\left\|D v_{n}\right\|^{2} \rightarrow 0$ and $\left\|\varphi_{n}\right\|^{2} \rightarrow 0$ in $L^{2}$. Thus, we have also $D u_{n} \rightarrow 0$, $v_{n} \rightarrow 0$ and, from (5.8) and (5.10), $u_{n} \rightarrow 0$ and $\phi_{n} \rightarrow 0$.

It only remains to see that $D \phi_{n} \rightarrow 0$. But, writing (5.11) again with the above information, we get $D^{2} \phi_{n} \rightarrow 0$.

This argument shows that $U_{n}$ can not be of unit norm, which finishes the proof of this lemma.

Lemma 5.3. Let $\mathcal{A}$ be the above defined matrix. Then condition (5.5) holds.

Proof: Suppose that the statement of the lemma is not true. Then, there is a sequence $\lambda_{n}$ with $\left|\lambda_{n}\right| \rightarrow \infty$ and a sequence of unit norm vectors in the domain of $\mathcal{A}, U_{n}=\left(u_{n}, v_{n}, \phi_{n}, \varphi_{n}\right)$, such that conditions (5.8)-(5.11) hold. Again $D v_{n}$ and $\varphi_{n}$ tend to zero and then $u_{n} \rightarrow 0$ and $\phi_{n} \rightarrow 0$ (in fact, $\lambda_{n} u_{n} \rightarrow 0$ and $\lambda_{n} \phi_{n} \rightarrow 0$ ).

Taking the inner product of (5.11) times $\phi_{n}$ and using (5.10) we get

$$
-\left\|\varphi_{n}\right\|^{2}+\frac{\gamma}{J}\left\|D \phi_{n}\right\|^{2} \rightarrow 0 \text { in } L^{2}
$$

Once again, this proves that $U_{n}$ can not be of unit norm.

Theorem 5.4. Let $(u, \phi)$ be a solution of the problem determined by (5.1), (3.2) and (3.3). Then $(u, \phi)$ decays exponentially.

Proof: The proof is a direct consequence of Lemmas 5.2 and 5.3.

Remark 5.5. We can make an spectral study of system (5.1). As in the proof of Theorem 4.1 , we obtain in this case a fourth degree polynomial $q(x)$ which coefficients depend on the parameters of the system. To be precise,

$$
\begin{array}{r}
q(x)=J \rho x^{4}+\sigma_{\nu}\left(J n^{2}+2 \rho\right) x^{3}+\left(J n^{2}(\mu+\sigma)+n^{2}\left(\gamma \rho+\sigma_{\nu}^{2}\right)+2 \rho \sigma\right) x^{2}+ \\
n^{2} \sigma_{\nu}\left(\gamma n^{2}+2(\mu+\sigma)\right) x+n^{2}\left(\gamma n^{2}(\mu+\sigma)+\sigma(2 \mu+\sigma)\right)
\end{array}
$$




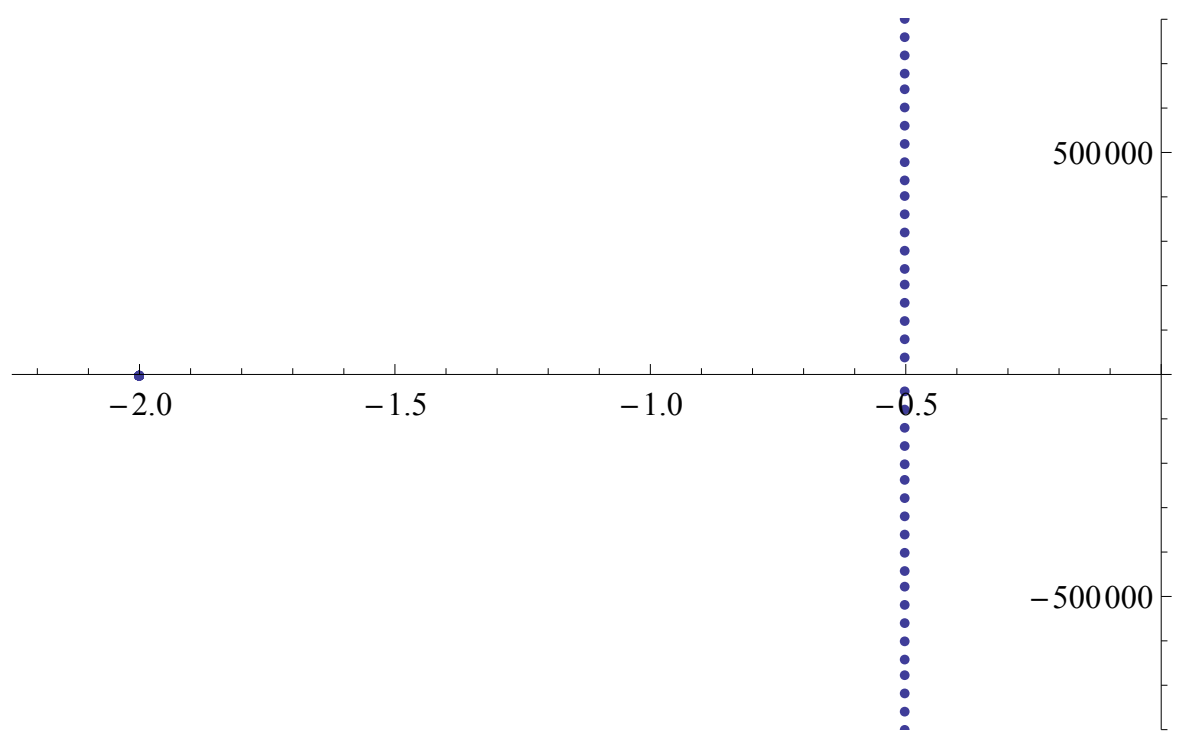

FiguRE 1. Root's behavior of $q_{P}(x)$.

Giving particular values (that satisfy the constitutive conditions) to the parameters of system (5.1) we get also a particular polynomial, $q_{P}(x)$. Take, for instance, $\rho=J=\gamma=\sigma=\mu=\sigma_{\nu}=$ 1. Then,

$$
q_{P}(x)=x^{4}+\left(n^{2}+2\right) x^{3}+\left(4 n^{2}+2\right) x^{2}+\left(n^{4}+4 n^{2}\right) x+2 n^{4}+3 n^{2} .
$$

Let us denote by $\alpha_{i n}$ for $i=1,2,3,4$ the roots of $q_{P}(x)$. It can be seen that two of them are real, suppose for instance $\alpha_{1, n}$ and $\alpha_{2, n}$ and the other two are conjugate complex numbers. If we take the limit when $n$ tends to infinity, we obtain that $\lim _{n \rightarrow \infty} \alpha_{1 n}=-2, \lim _{n \rightarrow \infty} \alpha_{2 n}=-\infty$ and $\lim _{n \rightarrow \infty} \Re \alpha_{3 n}=\lim _{n \rightarrow \infty} \Re \alpha_{4 n}=-\frac{1}{2}, \lim _{n \rightarrow \infty} \Im \alpha_{3 n}=\infty$ and $\lim _{n \rightarrow \infty} \Im \alpha_{4 n}=-\infty$. This fact leads us to think that the semigroup is not analytic.

In Figure 1 we have sketched some of the roots of $q_{P}(x)$ for different values of $n$ : we have computed 25 different polynomials beginning with $n=40000$ and taking an step of 40000 up to $n=1000000$.

\section{Non-LOCALization of SOlutions}

In this section, we will prove the non-localization of the solutions for the second and the third cases, that is, when we assume only viscopolarity and when we assume viscoelasticity and viscopolarity. If only viscoelasticity is assumed, the impossibility of localization of the solutions still holds, and the proof is, mutatis mutandis, the same as the one we present for the third case.

(A) Second case: viscopolarity.

We consider system (4.1) with boundary conditions (3.2) and initial conditions (3.3). To prove the impossibility of localization of the solutions, we recall that it is enough to show that, for the backward in time version of the problem, the only solution for null initial data is the null solution. The backward in time version of the system is

$$
\left\{\begin{array}{l}
\rho \ddot{u}=(\mu+\sigma) u_{x x}-\sigma \phi_{x} \\
J \ddot{\phi}=\gamma \phi_{x x}+\sigma u_{x}-2 \sigma \phi-\gamma_{\nu} \dot{\phi}_{x x}
\end{array}\right.
$$


First we look at the energy type functional

$$
\bar{E}_{1}(t)=\frac{1}{2} \int_{0}^{\pi}\left[\rho|\dot{u}|^{2}+J|\dot{\phi}|^{2}+(\mu+\sigma)\left|u_{x}\right|^{2}-2 \sigma u_{x} \phi+2 \sigma \phi^{2}+\gamma\left|\phi_{x}\right|^{2}\right] d x
$$

and we have

$$
\bar{E}_{1}^{\prime}(t)=\gamma_{\nu} \int_{0}^{\pi}\left|\dot{\phi}_{x}\right|^{2} d x
$$

Now, we define the following functional

$$
\bar{E}_{2}(t)=\frac{1}{2} \int_{0}^{\pi}\left[\rho|\dot{u}|^{2}+(\mu+\sigma)\left|u_{x}\right|^{2}-J|\dot{\phi}|^{2}-2 \sigma \phi^{2}-\gamma\left|\phi_{x}\right|^{2}\right] d x .
$$

Therefore,

$$
\bar{E}_{2}^{\prime}(t)=-\int_{0}^{\pi}\left[\sigma \phi_{x} \dot{u}+\sigma u_{x} \dot{\phi}\right] d x-\int_{0}^{\pi} \gamma_{\nu}\left|\dot{\phi}_{x}\right|^{2} d x .
$$

From system (6.1), we obtain the following identities, for a fixed $s \in(0,2 t)$ :

$$
\begin{gathered}
{\left[\rho \ddot{u}(s)-(\mu+\sigma) u_{x x}(s)+\sigma \phi_{x}(s)\right] \dot{u}(2 t-s)=0,} \\
{\left[\rho \ddot{u}(2 t-s)-(\mu+\sigma) u_{x x}(2 t-s)+\sigma \phi_{x}(2 t-s)\right] \dot{u}(s)=0,} \\
{\left[J \ddot{\phi}(s)-\gamma \phi_{x x}(s)-\sigma u_{x}(s)+2 \sigma \phi(s)+\gamma_{\nu} \dot{\phi}_{x x}(s)\right] \dot{\phi}(2 t-s)=0}
\end{gathered}
$$

and

$$
\left[J \ddot{\phi}(2 t-s)-\gamma \phi_{x x}(2 t-s)-\sigma u_{x}(2 t-s)+2 \sigma \phi(2 t-s)+\gamma_{\nu} \dot{\phi}_{x x}(2 t-s)\right] \dot{\phi}(s)=0 .
$$

Considering the equality $(6.6)-(6.7)+(6.8)-(6.9)=0$, integrating over $[0, \pi] \times[0, t]$ and taking into account the null data and the divergence theorem, we see that

$$
-\int_{0}^{\pi} J|\dot{\phi}|^{2} d x=\int_{0}^{\pi}\left[\rho|\dot{u}|^{2}-(\mu+\sigma)\left|u_{x}\right|^{2}+2 \sigma u_{x} \phi-2 \sigma \phi^{2}-\gamma\left|\phi_{x}\right|^{2}\right] d x .
$$

We use (6.10) in the expression (6.4) and we obtain

$$
\bar{E}_{2}(t)=\int_{0}^{\pi}\left[\rho|\dot{u}|^{2}+\sigma u_{x} \phi-2 \sigma \phi^{2}-\gamma\left|\phi_{x}\right|^{2}\right] d x .
$$

Clearly, there exists a positive constant $C_{5}$ such that

$$
\bar{E}_{2}^{\prime}(t) \leq C_{5} \int_{0}^{\pi}\left[|\dot{u}|^{2}+|\dot{\phi}|^{2}+\left|\phi_{x}\right|^{2}+\left|u_{x}\right|^{2}\right] d x-\gamma_{\nu} \int_{0}^{\pi}\left|\dot{\phi}_{x}\right|^{2} d x .
$$

We note that

$$
\int_{0}^{\pi} J \ddot{\phi} \phi d x=\int_{0}^{\pi} J \frac{d}{d t}(\phi \dot{\phi}) d x-\int_{0}^{\pi} J|\dot{\phi}|^{2} d x
$$

Therefore,

$$
\begin{aligned}
& \int_{0}^{\pi} J \frac{d}{d t}(\phi \dot{\phi}) d x-\int_{0}^{\pi} J|\dot{\phi}|^{2} d x= \\
& -\int_{0}^{\pi} \gamma\left|\phi_{x}\right|^{2} d x+\int_{0}^{\pi} \sigma u_{x} \phi d x-2 \sigma \int_{0}^{\pi} \phi^{2} d x+\frac{\gamma_{\nu}}{2} \int_{0}^{\pi} \frac{d}{d t}\left|\phi_{x}\right|^{2} d x .
\end{aligned}
$$


It follows that

$$
\frac{d}{d t} \int_{0}^{\pi}\left[\frac{\gamma_{\nu}}{2}\left|\phi_{x}\right|^{2}-J \phi \dot{\phi}\right] d x=\int_{0}^{\pi}\left[\gamma\left|\phi_{x}\right|^{2} d x-\sigma \phi u_{x} d x+2 \sigma \phi^{2}-J|\dot{\phi}|^{2}\right] d x .
$$

Let us consider the functional

$$
\bar{E}_{0}(t)=\varepsilon \bar{E}_{1}(t)+\bar{E}_{2}(t)+\lambda \bar{E}_{3}(t)
$$

where

$$
\bar{E}_{3}(t)=\int_{0}^{\pi}\left[\frac{\gamma_{\nu}}{2}\left|\phi_{x}\right|^{2}-J \phi \dot{\phi}\right] d x
$$

and $\varepsilon$ is a positive real number as small as we want and $\lambda$ is also a positive real number as large as necessary. Direct calculations give

$$
\bar{E}_{3}^{\prime}(t)=\int_{0}^{\pi}\left[\gamma\left|\phi_{x}\right|^{2}+\sigma \phi_{x} u+2 \sigma \phi^{2}-J|\dot{\phi}|^{2}\right] d x
$$

Here, if $\lambda$ is large enough and $t$ is sufficiently small, there exists a positive constant $C_{6}$ such that

$$
\bar{E}_{0}(t) \geq C_{6} \int_{0}^{\pi}\left[|\dot{u}|^{2}+|\dot{\phi}|^{2}+\left|u_{x}\right|^{2}+|\phi|^{2}+\left|\phi_{x}\right|^{2}\right] d x-\lambda \int_{0}^{\pi} J \phi \dot{\phi} d x .
$$

Let us consider the following function

$$
\overline{\mathcal{E}}(t)=\int_{0}^{t} \bar{E}_{0}(s) d s
$$

for $t \leq t_{0}$. Then,

$$
\overline{\mathcal{E}}(t) \geq C_{6} \int_{0}^{t} \int_{0}^{\pi}\left[|\dot{u}|^{2}+|\dot{\phi}|^{2}+\left|u_{x}\right|^{2}+|\phi|^{2}+\left|\phi_{x}\right|^{2}\right] d x d s-\lambda \int_{0}^{t} \int_{0}^{\pi} J \phi \dot{\phi} d x d s .
$$

Furthermore, from the Hölder and Poincaré inequalities, we get

$$
\begin{aligned}
-\lambda \int_{0}^{t} \int_{0}^{\pi} J \phi \dot{\phi} d x d s & \geq-\lambda J\left(\int_{0}^{t} \int_{0}^{\pi}|\phi|^{2} d x d s\right)^{1 / 2}\left(\int_{0}^{t} \int_{0}^{\pi}|\dot{\phi}|^{2} d x d s\right)^{1 / 2} \\
& \geq-\lambda J \frac{2 t}{\pi} \int_{0}^{t} \int_{0}^{\pi}|\dot{\phi}|^{2} d x d s
\end{aligned}
$$

We note that, for fixed but large $\lambda$ and small $t$, the integral of $C_{6}|\dot{\phi}|^{2}$ controls the integral of $-\lambda J \frac{2 t}{\pi}|\dot{\phi}|^{2}$. So, there exists a positive constant $C_{7}$ such that

$$
\overline{\mathcal{E}}(t) \geq C_{7} \int_{0}^{t} \int_{0}^{\pi}\left[|\dot{u}|^{2}+|\dot{\phi}|^{2}+\left|u_{x}\right|^{2}+|\phi|^{2}+\left|\phi_{x}\right|^{2}\right] d x d s
$$

for $t$ sufficiently small. From the initial null conditions, we have

$$
\overline{\mathcal{E}}^{\prime}(t)=\int_{0}^{t} \bar{E}^{\prime}(s) d s .
$$


Thus, from (6.3), (6.12) and (6.18) we obtain

$$
\begin{aligned}
\overline{\mathcal{E}}^{\prime}(t) \leq & \varepsilon \gamma_{\nu} \int_{0}^{t} \int_{0}^{\pi}\left|\dot{\phi}_{x}\right|^{2} d x d s \\
& +C_{5} \int_{0}^{t} \int_{0}^{\pi}\left[|\dot{u}|^{2}+|\dot{\phi}|^{2}+\left|\phi_{x}\right|^{2}+\left|u_{x}\right|^{2}\right] d x d s-\gamma_{\nu} \int_{0}^{t} \int_{0}^{\pi}\left|\dot{\phi}_{x}\right|^{2} d x d s \\
& +\lambda \int_{0}^{t} \int_{0}^{\pi}\left[\gamma\left|\phi_{x}\right|^{2}+\sigma \phi_{x} u+2 \sigma \phi^{2}\right] d x d s .
\end{aligned}
$$

If $\varepsilon<1$, the term $\varepsilon \gamma_{\nu}\left|\dot{\phi}_{x}\right|^{2}$ is controlled by $-\gamma_{\nu}\left|\dot{\phi}_{x}\right|^{2}$. Then, it follows that

$$
\overline{\mathcal{E}}^{\prime}(t) \leq K_{2} \int_{0}^{t} \int_{0}^{\pi}\left[|\dot{\phi}|^{2}+|\dot{u}|^{2}+\left|u_{x}\right|^{2}+|\phi|^{2}+\left|\phi_{x}\right|^{2}\right] d x d s,
$$

for some $K_{2}>0$. Therefore, there exists a positive constant $M$ such that

$$
\overline{\mathcal{E}}^{\prime}(t) \leq M \overline{\mathcal{E}}(t)
$$

Hence,

$$
\overline{\mathcal{E}}(t) \leq \overline{\mathcal{E}}(0) e^{M t}, \quad t \leq t_{0} .
$$

For null initial conditions, the estimate (6.28) gives $\mathcal{E}(t) \leq 0$, for $t \leq t_{0}$. We can repeat our arguments for $t_{0} \leq t \leq 2 t_{0}$ and so on. From the definition of $\overline{\mathcal{E}}(t)$ it follows that the solution is identically null for all positive time and consequently the non-localization is proved.

(B) Third case: viscoelasticity and viscopolarity.

We consider system (5.1) with boundary conditions (3.2) and initial conditions (3.3). The backward in time version of our system is

$$
\left\{\begin{array}{l}
\rho \ddot{u}=(\mu+\sigma) u_{x x}-\sigma \phi_{x}-\sigma_{\nu} \dot{u}_{x x}+\sigma_{\nu} \dot{\phi}_{x} \\
J \ddot{\phi}=\gamma \phi_{x x}+\sigma u_{x}-2 \sigma \phi-\sigma_{\nu} \dot{u}_{x}+2 \sigma_{\nu} \dot{\phi}
\end{array}\right.
$$

First, we consider the energy function

$$
E_{1}(t)=\frac{1}{2} \int_{0}^{\pi}\left[\rho|\dot{u}|^{2}+J|\dot{\phi}|^{2}+(\mu+\sigma)\left|u_{x}\right|^{2}-2 \sigma u_{x} \phi+2 \sigma \phi^{2}+\gamma\left|\phi_{x}\right|^{2}\right] d x .
$$

Direct computations give

$$
E_{1}^{\prime}(t)=\int_{0}^{\pi}\left[\sigma_{\nu}\left(\left|\dot{u}_{x}\right|^{2}-2 \dot{\phi} \dot{u}_{x}+|\dot{\phi}|^{2}\right)+\sigma_{\nu}|\dot{\phi}|^{2}\right] d x
$$

It is clear that there exists a positive constant $C_{1}$ such that

$$
E_{1}^{\prime}(t) \leq C_{1} \int_{0}^{\pi}\left(\left|\dot{u}_{x}\right|^{2}+|\dot{\phi}|^{2}\right) d x .
$$

Now we define another functional

$$
E_{2}(t)=\frac{1}{2} \int_{0}^{\pi}\left[J|\dot{\phi}|^{2}+2 \sigma \phi^{2}+\gamma\left|\phi_{x}\right|^{2}-\rho|\dot{u}|^{2}-(\mu+\sigma)\left|u_{x}\right|^{2}\right] d x .
$$

Therefore,

$$
E_{2}^{\prime}(t)=\int_{0}^{\pi}\left[\sigma u_{x} \dot{\phi}+2 \sigma_{\nu}|\dot{\phi}|^{2}-\sigma \phi \dot{u}_{x}-\sigma_{\nu}\left|\dot{u}_{x}\right|^{2}\right] d x
$$


On the other hand, from system (6.29), we can establish the following identities for a fixed $s \in(0,2 t)$ :

$$
\begin{gathered}
{\left[\rho \ddot{u}(s)-(\mu+\sigma) u_{x x}(s)+\sigma \phi_{x}(s)+\sigma_{\nu} \dot{u}_{x x}(s)-\sigma_{\nu} \dot{\phi}_{x}(s)\right] \dot{u}(2 t-s)=0,} \\
{\left[\rho \ddot{u}(2 t-s)-(\mu+\sigma) u_{x x}(2 t-s)+\sigma \phi_{x}(2 t-s)+\sigma_{\nu} \dot{u}_{x x}(2 t-s)\right.} \\
\left.-\sigma_{\nu} \dot{\phi}_{x}(2 t-s)\right] \dot{u}(s)=0 \\
{\left[J \ddot{\phi}(s)-\gamma \phi_{x x}(s)-\sigma u_{x}(s)+2 \sigma \phi(s)+\sigma_{\nu} \dot{u}_{x}(s)-2 \sigma_{\nu} \dot{\phi}(s)\right] \dot{\phi}(2 t-s)=0}
\end{gathered}
$$

and

$$
\begin{array}{r}
{\left[J \ddot{\phi}(2 t-s)-\gamma \phi_{x x}(2 t-s)-\sigma u_{x}(2 t-s)+2 \sigma \phi(2 t-s)+\sigma_{\nu} \dot{u}_{x}(2 t-s)\right.} \\
\left.-2 \sigma_{\nu} \dot{\phi}(2 t-s)\right] \dot{\phi}(s)=0 .
\end{array}
$$

Considering the following equality $(6.35)-(6.36)+(6.37)-(6.38)=0$, integrating over $[0, \pi] \times[0, t]$ and taking into account the null data and the divergence theorem, we obtain that

$$
\int_{0}^{\pi}\left[\rho|\dot{u}|^{2}+J|\dot{\phi}|^{2}\right] d x=\int_{0}^{\pi}\left[(\mu+\sigma)\left|u_{x}\right|^{2}-2 \sigma u_{x} \phi+2 \sigma \phi^{2}+\gamma\left|\phi_{x}\right|^{2}\right] d x .
$$

So,

$$
-\int_{0}^{\pi} \rho|\dot{u}|^{2} d x=\int_{0}^{\pi}\left[J|\dot{\phi}|^{2}-(\mu+\sigma)\left|u_{x}\right|^{2}+2 \sigma u_{x} \phi-2 \sigma \phi^{2}-\gamma\left|\phi_{x}\right|^{2}\right] d x .
$$

Now, if we introduce (6.40) into (6.33), we get

$$
E_{2}(t)=\int_{0}^{\pi}\left[J|\dot{\phi}|^{2}+\sigma u_{x} \phi-(\mu+\sigma)\left|u_{x}\right|^{2}\right] d x .
$$

Clearly, from (6.34) and the A-G inequality, there exists a positive constant $C_{2}$ such that

$$
E_{2}^{\prime}(t) \leq C_{2} \int_{0}^{\pi}\left[\left|u_{x}\right|^{2}+|\phi|^{2}+|\dot{\phi}|^{2}\right] d x-\frac{\sigma_{\nu}}{2} \int_{0}^{\pi}\left|\dot{u}_{x}\right|^{2} d x
$$

We note that

$$
\int_{0}^{\pi} \rho \ddot{u} u d x=\int_{0}^{\pi} \rho \frac{d}{d t}(u \dot{u}) d x-\int_{0}^{\pi} \rho|\dot{u}|^{2} d x .
$$

We multiply the first equation of the system (6.29) by $u$ and we take into account (6.43). Therefore, we obtain

$$
\begin{aligned}
& \int_{0}^{\pi} \rho \frac{d}{d t}(u \dot{u}) d x-\int_{0}^{\pi} \rho|\dot{u}|^{2} d x= \\
& -\int_{0}^{\pi}(\mu+\sigma)\left|u_{x}\right|^{2} d x-\int_{0}^{\pi} \sigma \phi_{x} u d x+\int_{0}^{\pi} \frac{\sigma_{\nu}}{2} \frac{d}{d t}\left|u_{x}\right|^{2} d x-\int_{0}^{\pi} \sigma_{\nu} \dot{\phi} u_{x} d x .
\end{aligned}
$$

And thus,

$$
\frac{d}{d t} \int_{0}^{\pi}\left[\frac{\sigma_{\nu}}{2}\left|u_{x}\right|^{2}-\rho u \dot{u}\right] d x=\int_{0}^{\pi}\left[(\mu+\sigma)\left|u_{x}\right|^{2} d x+\sigma \phi_{x} u d x+\sigma_{\nu} \dot{\phi} u_{x} d x-\rho|\dot{u}|^{2}\right] d x
$$


Let us consider the functional

$$
E_{0}(t)=\varepsilon E_{1}(t)+E_{2}(t)+\lambda E_{3}(t)
$$

where

$$
E_{3}(t)=\int_{0}^{\pi}\left[\frac{\sigma_{\nu}}{2}\left|u_{x}\right|^{2}-\rho u \dot{u}\right] d x
$$

and, as in the previous case, $\varepsilon>0$ is a positive real number as small as we want and $\lambda$ is again a positive real number as large as necessary. Easy computations give

$$
E_{3}^{\prime}(t)=\int_{0}^{\pi}\left[(\mu+\sigma)\left|u_{x}\right|^{2}+\sigma \phi_{x} u+\sigma_{\nu} \dot{\phi} u_{x}-\rho|\dot{u}|^{2}\right] d x .
$$

We note that, if $\lambda$ is sufficiently large and $t$ is small enough, there exists a positive constant $C_{3}$ such that

$$
E_{0}(t) \geq C_{3} \int_{0}^{\pi}\left[|\dot{u}|^{2}+|\dot{\phi}|^{2}+\left|u_{x}\right|^{2}+|\phi|^{2}+\left|\phi_{x}\right|^{2}\right] d x-\lambda \int_{0}^{\pi} \rho u \dot{u} d x .
$$

Now we consider the following function

$$
\mathcal{E}(t)=\int_{0}^{t} E_{0}(s) d s
$$

for $t \leq t_{0}$. Therefore,

$$
\mathcal{E}(t) \geq C_{3} \int_{0}^{t} \int_{0}^{\pi}\left[|\dot{u}|^{2}+|\dot{\phi}|^{2}+\left|u_{x}\right|^{2}+|\phi|^{2}+\left|\phi_{x}\right|^{2}\right] d x d s-\lambda \int_{0}^{t} \int_{0}^{\pi} \rho u \dot{u} d x d s .
$$

On the other hand, from the Hölder and Poincaré inequalities, we get

$$
\begin{aligned}
-\lambda \int_{0}^{t} \int_{0}^{\pi} \rho u \dot{u} d x d s & \geq-\lambda \rho\left(\int_{0}^{t} \int_{0}^{\pi}|u|^{2} d x d s\right)^{1 / 2}\left(\int_{0}^{t} \int_{0}^{\pi}|\dot{u}|^{2} d x d s\right)^{1 / 2} \\
& \geq-\lambda \rho \frac{2 t}{\pi} \int_{0}^{t} \int_{0}^{\pi}|\dot{u}|^{2} d x d s .
\end{aligned}
$$

It is worth noting that, for fixed but large $\lambda$ and small $t$, the integral of $C_{3}|\dot{u}|^{2}$ controls the integral of $-\lambda \rho \frac{2 t}{\pi}|\dot{u}|^{2}$. So, there exists a positive constant $C_{4}$ such that

$$
\mathcal{E}(t) \geq C_{4} \int_{0}^{t} \int_{0}^{\pi}\left[|\dot{u}|^{2}+|\dot{\phi}|^{2}+\left|u_{x}\right|^{2}+|\phi|^{2}+\left|\phi_{x}\right|^{2}\right] d x d s,
$$

for $t$ sufficiently small. Moreover,

$$
\mathcal{E}^{\prime}(t)=\int_{0}^{t} E^{\prime}(s) d s
$$

because the initial conditions are null. Hence, from (6.32), (6.42) and (6.48) we obtain

$$
\begin{aligned}
\mathcal{E}^{\prime}(t) \leq & \varepsilon C_{1} \int_{0}^{t} \int_{0}^{\pi}\left(\left|\dot{u}_{x}\right|^{2}+|\dot{\phi}|^{2}\right) d x d s \\
& +C_{2} \int_{0}^{t} \int_{0}^{\pi}\left[\left|u_{x}\right|^{2}+|\phi|^{2}+|\dot{\phi}|^{2}\right] d x d s-\frac{\sigma_{\nu}}{2} \int_{0}^{t} \int_{0}^{\pi}\left|\dot{u}_{x}\right|^{2} d x d s \\
& +\lambda \int_{0}^{t} \int_{0}^{\pi}\left[(\mu+\sigma)\left|u_{x}\right|^{2}+\sigma \phi_{x} u+\sigma_{\nu} \dot{\phi} u_{x}\right] d x d s .
\end{aligned}
$$


We choose $\varepsilon$ sufficiently small so that $\varepsilon C_{1}<\frac{\sigma_{\nu}}{2}$ in order to control the integral of $\varepsilon C_{1}\left|\dot{u}_{x}\right|^{2}$ by the integral of $\frac{-\sigma_{\nu}}{2}\left|\dot{u}_{x}\right|^{2}$. Then, it follows that

$$
\mathcal{E}^{\prime}(t) \leq K_{1} \int_{0}^{t} \int_{0}^{\pi}\left[|\dot{u}|+|\dot{\phi}|^{2}+\left|u_{x}\right|^{2}+|\phi|^{2}+\left|\phi_{x}\right|^{2}\right] d x d s,
$$

for some $K_{1}>0$. Thus, there exists a positive constant $K$ such that

$$
\mathcal{E}^{\prime}(t) \leq K \mathcal{E}(t)
$$

and the result follows.

It is worth noting that the impossibility of localization of the solutions for the first case (viscoelasticity) can be proved in a similar way.

\section{Conclusions}

We analyzed isotropic micropolar viscoelastic materials whose points are allowed to rotate in one direction (the one-dimensional case). We consider three different dissipation mechanisms:

(1) First we introduce only viscoelasticity (viscosity effects at the macrostructure of the material) and we see that the resulting mathematical system is a particular case of a previously studied system and whose solutions decay in a slow way with respect to the time. In fact, it can be seen that a polynomial rate of decay can be found for them.

(2) Second we introduce only viscopolarity (viscosity effects at the microstructure of the material) and we prove that the solutions of the resulting system decay also in a slow way and following a polynomial rate.

(3) Third we introduce viscoelasticity and also viscopolarity (viscosity effects in both structures of the material) and we prove that, therefore, the solutions of the resulting system decay exponentially, that is, they are exponentially stable.

In all three cases, the impossibility of localization of solutions is given. To prove the exponential decay we have used semigroup arguments and to show the polynomial rate of decay we have used energy methods.

\section{ACKNOWLedgments}

The investigation reported in this paper is supported by the project "Análisis Matemático de las Ecuaciones en Derivadas Parciales de la Termomecánica" (MTM2013-42004-P) of the Spanish Ministry of Economy and Competitiveness.

\section{REFERENCES}

[1] Alves MS, Muñoz Rivera JE, Quintanilla R (2009) Exponential decay in a thermoelastic mixture of solids. Int J Solids Structures 46 no.7-8:1659-1666

[2] Alves MS, Muñoz Rivera JE, Sepúlveda M, Villagrán OV (2009) Exponential stability in thermoviscoelastic mixtures of solids. Int J Solids Structures 46 no.24:4151-4162

[3] Alves MS, Muñoz Rivera JE, Sepúlveda M, Villagrán OV (2009) Analyticity of semigroups associates with thermoeviscoelastic mixtures of solid. J Thermal Stresses 32:986-1004

[4] Casas PS, Quintanilla R (2005) Exponential stability in thermoelasticity with microtemperatures. Int J Eng Sci 43:33-47 
[5] Casas PS, Quintanilla R (2005) Exponential decay in one-dimensional porous-themoelasticity. Mech Res Comm 32:652-658

[6] Elangovan S, Altan BS, Odegard GM (2008) An Elastic Micropolar Mixture Theory for Predicting Elastic Properties of Cellular Materials. Mechanics of Materials 40(7):602-615

[7] Eringen AC (1967) Linear theory of micropolar viscoelasticity. Int J Eng Sci 5 no.2:191-204

[8] Erigen AC (1999) Microcontinuum Field Theories. I: Foundations and Solids. Springer-Verlag, New York.

[9] Fatemi J, Van Keulen, F, Onck PR, (2002) Generalized Continuum Theories: Application to Stress Analysis in Bone, Meccanica 37:385-396

[10] Fernández-Sare H, Muñoz Rivera JE, Quintanilla R (2010) Decay of solutions in nonsimple thermoelastic bars. Int J Eng Sci 48 no.11:1233-1241

[11] Glowinski P, Lada A (2009) Stabilization of elasticity-viscoporosity system by linear boundary feedback. Math Methos Appl Sci 32:702-722

[12] Han ZJ, Xu GQ (2012) Exponential decay in non-uniform porous-thermo-elasticity model of Lord-Shulman type. Discrete and Continuous Dynam. Systems - B 17:57-77

[13] Ieşan D (2004) Thermoelastic Models of Continua. Springer

[14] Kordolemis A, Giannakopoulos AE (2014) Micropolar 2D Elastic Cables with Applications to Smart Cables and Textiles. J Eng Mech, 140(10), 04014079

[15] Lazzari B, Nibbi R (2009) On the influence of a dissipative boundary on the energy decay for a porous elastic solid. Mech Res Comm 36:581-586

[16] Leseduarte MC, Magaña A, Quintanilla R (2010) On the time decay of solutions in porous-thermo-elasticity of type II. Discrete and Continuous Dynam. Systems - B 13:375-391

[17] Liu Z, Zheng S, (1999) Semigroups associated with dissipative systems. Chapman and Hall/CRC

[18] Magaña A, Quintanilla R (2006) On the exponential decay of solutions in one-dimensional generalized porousthermo-elasticity. Asym Anal 49:173-187

[19] Magaña A, Quintanilla R (2006) On the time decay of solutions in one-dimensional theories of porous materials. Int J Solids Structures 43:3414-3427

[20] Magaña A, Quintanilla R (2007) On the time decay of solutions in porous elasticity with quasistatic microvoids. J Math Anal Appl 331:617-630

[21] Magaña A, Quintanilla R (2014) On the uniqueness and analyticity of solutions in micropolar thermoviscoelasticity. J Math Anal Appl 412:109-120

[22] Messaoudi SA, Fareh A (2011) General decay for a porous thermoelastic system with memory: The case of equal speeds. Nonlinear Analysis 74:6895-6906

[23] Muñoz Rivera JE, Quintanilla R (2008) On the time polynomial decay in elastic solids with voids. J Math Anal Appl 338:1296-1309

[24] Nicasise S, Valein J (2012) Stabilization of non-homogeneous elastic materials with voids. J Math Anal Appl $387: 1061-1087$

[25] Nikogosyan GS, Sargsyan SH, Thin shells on the basis of asymmetric theory of elasticity (2006) Shell Structures: Theory and Applications. Taylor \& Francis Group, London 153-156

[26] Pamplona PX, Muñoz Rivera JE, Quintanilla R (2009) Stabilization in elastic solids with voids. J Math Anal Appl 350:37-49

[27] Pamplona PX, Muñoz Rivera JE, Quintanilla R (2011) On the decay of solutiosn for porous-elastic systems with history. J Math Anal Appl 379:251-266

[28] Pamplona PX, Muñoz Rivera JE, Quintanilla R (2011) On uniqueness and analyticity in thermoviscoelastic solids with voids. J Appl Analysis Computation 1:682-705

[29] Pata V, Quintanilla R (2010) On the decay of solutions in nonsimple elastic solids with memory. J Math Anal Appl 363:19-28

[30] Quintanilla R (2003) Slow decay for one-dimensional porous dissipation elasticity. Appl Math Lett 16:487-491

[31] Quintanilla R (2005) Existence and exponential decay in the linear theory of viscoelastic mixtures. Eur J Mech A Solids 24 no.2:311-324

[32] Quintanilla R (2005) Exponential decay in mixtures with localized dissipative term. Appl Math Lett 18 no. $12: 1381-1388$

[33] Soufyane A (2008) Energy decay for porous-thermo-elasticity systems of memory type. Applicable Analysis $87: 451-464$ 